Case Report

\title{
Chronic Lymphocytic Leukemia with Translocation (2;14)(p16;q32): A Case Report and Review of the Literature
}

\author{
Francisco Socola, ${ }^{1}$ Giovanni Insuasti-Beltran, ${ }^{2}$ Rodolfo Henrich Lobo, ${ }^{2}$ \\ Shebli Atrash, ${ }^{1}$ and Appalanaidu Sasapu ${ }^{1}$ \\ ${ }^{1}$ Department of Medicine, Division of Hematology Oncology, University of Arkansas for Medical Science, Little Rock, AR, USA \\ ${ }^{2}$ Department of Hematopathology/Molecular Genetics Pathology, University of Arkansas for Medical Sciences, Little Rock, AR, USA
}

Correspondence should be addressed to Francisco Socola; fasocola@uams.edu

Received 26 July 2016; Accepted 13 October 2016

Academic Editor: Junya Kuroda

Copyright (C) 2016 Francisco Socola et al. This is an open access article distributed under the Creative Commons Attribution License, which permits unrestricted use, distribution, and reproduction in any medium, provided the original work is properly cited.

We report the case of a young African American male with no significant past medical history presenting with low back and bilateral leg pain; presenting $\mathrm{CBC}$ and chemistries revealed elevated white blood cell count of 250,000, with anemia ( $\mathrm{Hb}$ $6.8 \mathrm{~g} / \mathrm{dL}$ ) and thrombocytopenia (platelets $9 \mathrm{~K} / \mu \mathrm{L}$ ), and elevated $\mathrm{LDH}, 1008$. Physical examination findings were notable for diffuse lymphadenopathy and lower extremity skin nodules. Interestingly the bone marrow biopsy revealed involvement by CLL/SLL with translocation $(2 ; 14)(\mathrm{p} 16 ; \mathrm{q} 32)$ and trisomy 12 . The patient was treated with fludarabine-based chemotherapy and steroids for CLLrelated ITP with excellent response. After three cycles of chemotherapy, all the enlarged lymph nodes and skin nodules disappeared, and patient had achieved complete hematologic response. In this paper we also reviewed the available literature of CLL patients with translocation $(2 ; 14)$.

\section{Introduction}

Chronic lymphocytic leukemia/small lymphocytic lymphoma (CLL/SLL) is the most common type of leukemia in adults, and it is characterized for having chromosomal abnormalities in up to $80 \%$ of patients. Among them, deletions of 11q, 13q, 17p, and trisomy 12 have a known prognostic value and play an important role in CLL pathogenesis and evolution, determining patient's outcomes and therapeutic strategies. Many B-cell lymphomas are characterized by chromosomal translocations that involve the immunoglobulin genes; however, chromosome translocations including $I G H$ rearrangement on $14 \mathrm{q} 32$ were relatively infrequent in CLL/SLL, with a frequency of $4 \%$ [1].

The B-cell CLL/lymphoma 11A (BCL11A) gene is located in chromosome 2 p16.1 and encodes a zinc finger protein that interacts directly with BCL6, a known human B-cell protooncogene that serves a crucial role in lymphocyte development [1]. It is expressed at low levels, mainly in the nuclei of B cells in the germinal centers and mantle zones, a subset of cells in the interfollicular areas of tonsil and lymph nodes, and in the splenic marginal zones [2, 3]. The overexpression of the BCL11A gene has been described in 18 cases of CLL/SLL, invariably associated with the $t(2,14)$ translocation [4-10]. This translocation creates an abnormal fusion between the BCL11A (2p16.1) and immunoglobulin heavy chain $(I G H)$ gene $(14 \mathrm{q} 32)$, resulting in uncontrolled overexpression of the BCL11A protein and therefore favoring leukemogenesis.

In regard to the BCL11A gene location, it was initially mapped to chromosome $2 \mathrm{p} 13$, where some cases of lymphoblastic leukemia had shown chromosomal translocations [11]. Subsequently, Menzel et al. stated the location of this gene as $2 \mathrm{p} 15$ [12]. It is currently accepted that BCL11A gene maps to chromosome 2 p16.1 [1].

\section{Case Presentation}

A 49-year-old African American man with history of chronic back pain presented to the ER with three-week history of worsening lower back and bilateral leg pain. He was 


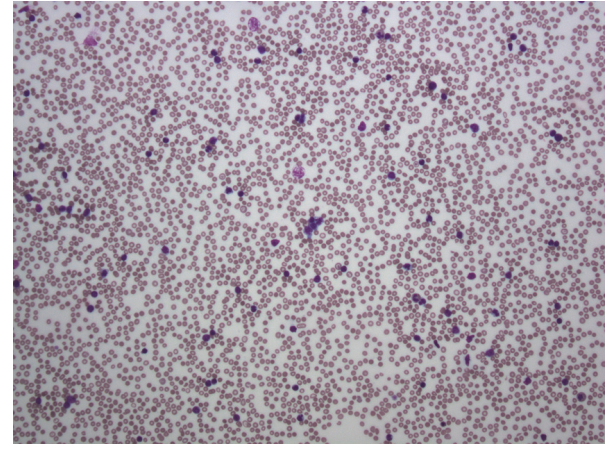

FIgURE 1: Lymphocytosis in the peripheral blood.

afebrile and normotensive on presentation with physical examination findings that were notable for tenderness in the lower lumbar spine, nontender enlarged lymph nodes in the cervical, supraclavicular, axillary and inguinal regions, multiple subcutaneous nodules in the skin of the proximal lower extremities; the abdomen was only positive for mild splenomegaly with normal liver size. In the neurologic exam, the motor strength was preserved in all the extremities with normal deep tendon reflexes.

Presenting $\mathrm{CBC}$ and chemistries showed white blood cell count (WBC) of $250 \mathrm{~K} / \mu \mathrm{L}$, with $1 \%$ neutrophils and 99\% lymphocytes, hemoglobin, $6.8 \mathrm{~g} / \mathrm{dL}$, and platelet count of $9 \mathrm{~K} / \mu \mathrm{L}$. BMP, LFTs, DIC panel, HIV, and viral hepatitis panel were normal. Tumor lysis panel was only significant for elevated LDH 1008. Peripheral smear showed marked mature lymphocytosis, composed of medium sized cells with mature clumped chromatin, round nuclear contours, moderate cytoplasm, severe thrombocytopenia and hypochromic, and normocytic anemia (Figure 1). Flow cytometry identified $85 \%$ of total events as CD19 and CD20 positive Bcells coexpressing CD5, CD23 (heterogeneous), CD200, and kappa light chain. CD38 was expressed in $>30 \%$ of clonal Bcells. They were negative for CD10, FMC7, and CD34. CT scans revealed cervical lymphadenopathy involving levels I through V bilaterally, as well as extensive lymphadenopathy in the bilateral axilla, inguinal, and external iliac chains. MRI of the spine revealed diffusely abnormal T1 marrow signal, indicative of a marrow replacement by leukemia; no spinal canal stenosis or nerve root impingement was found.

Bone marrow biopsy and aspirate showed a markedly hypercellular marrow for age ( $95 \%$ cellularity), essentially replaced by small, mature-appearing lymphoid cells in a diffuse pattern of distribution. The disease burden was estimated at about $90-95 \%$ of marrow space, with severely decreased erythropoiesis and granulopoiesis. Megakaryocytes were increased in number and showed adequate morphology (Figures 2, 3, and 4). FISH revealed trisomy 12 and gain of $14 \mathrm{q} 32(\mathrm{IGH})$ in $67 \%$ and $27 \%$ of analyzed nuclei, respectively, as well as gains of 6q, 11q22.3 (ATM), 13q, and 17p13.1 (TP53) in a low number of nuclei $(<10 \%)$. Conventional cytogenetics demonstrated an abnormal male karyotype: $47, \mathrm{XY}, \mathrm{t}(2 ; 14)(\mathrm{p} 16 ; \mathrm{q} 32),+12[\mathrm{cp} 11] / 46, \mathrm{XY}[1]$, significant for the presence of the $t(2 ; 14)$ translocation (BCL11A-IGH) and trisomy 12 (Figure 5). Unfortunately insufficient material was

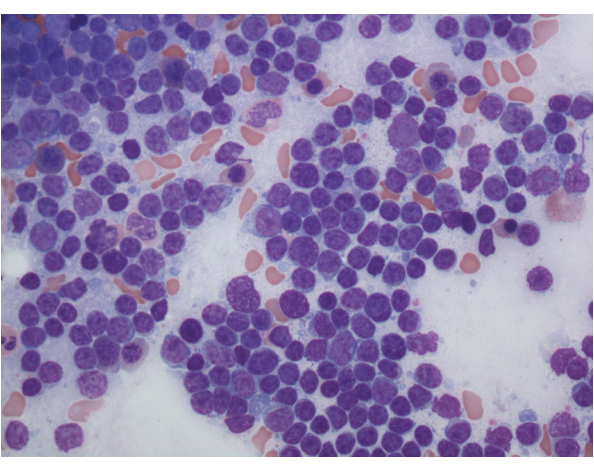

FIGURE 2: Bone marrow aspirate depicts the marked increase in lymphocytes. They are uniformly small with mature chromatin, scant cytoplasm, and inconspicuous nucleoli.

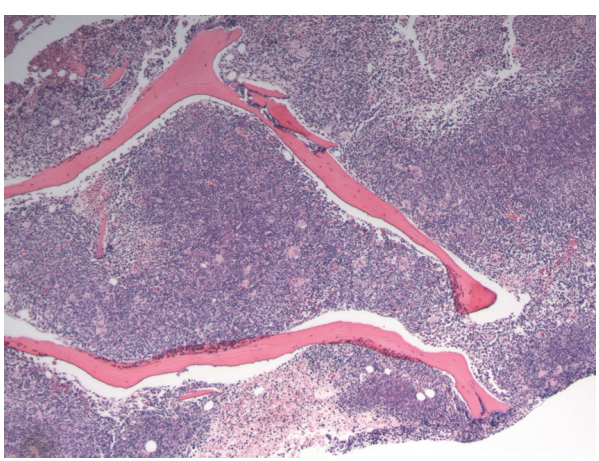

Figure 3: Diffuse involvement of the bone marrow by CLL as shown on this core biopsy specimen.

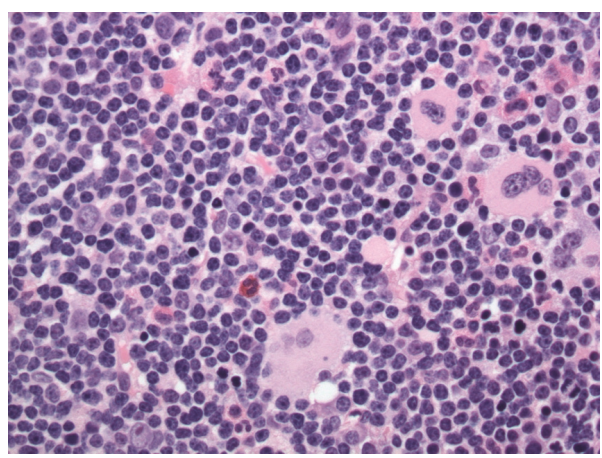

FIGURE 4: High-power view of the biopsy depicting the markedly increased number of small, mature-appearing lymphoid cells.

available to perform specific FISH to further demonstrate the presence of the $B C L 11 A-I G H$ translocation, but analysis of the oncogenes present in chromosome 2 p16 renders BCL11A as the only potential candidate with demonstrated oncogenic capacity in CLL/SLL [13]. IGHV gene mutation analysis was reported as unmutated.

The patient was diagnosed with chronic lymphocytic leukemia/small lymphocytic lymphoma (CLL/SLL) and autoimmune thrombocytopenic purpura (ITP). He was started on IV dexamethasone and after two days, he was treated with fludarabine, cyclophosphamide, and rituximab, 


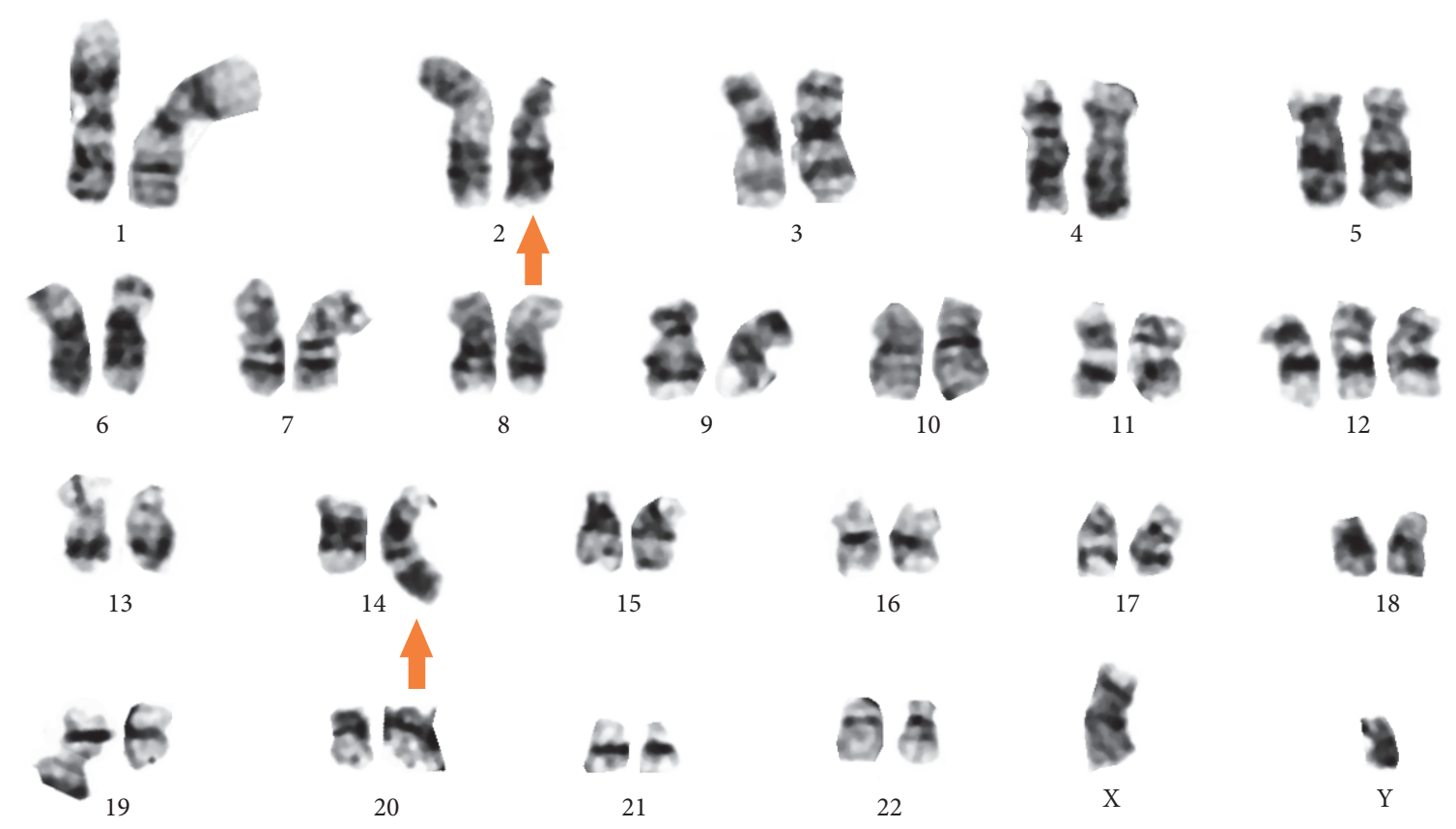

FIgURE 5: Cytogenetics showing translocation (2;14)(p16;q32). Orange arrow.

with rapid improvement of the cell counts; after 2 weeks, the WBC decreased to $12 \mathrm{~K} / \mu \mathrm{L} ; \mathrm{Hb}, 9.1 \mathrm{~g} / \mathrm{dL}$; and platelet count, $58 \mathrm{~K} / \mu \mathrm{L}$. At the time of the last clinic appointment, the patient had had 4 cycles of FCR and he was asymptomatic and had achieved complete hematologic response, and all the enlarged lymph nodes and skin nodules disappeared.

\section{Discussion}

We performed a review of the available literature and were able to find 19 cases reported; briefly we will summarize them:

Yin et al. described 6 CLL cases with $\mathrm{t}(2 ; 14)(\mathrm{p} 16 ; \mathrm{q} 32)$; these patients had an average age of 49.6 years. All of them had marrow involvement, 4 had absolute lymphocytosis, 4 had lymphadenopathy, and 3 of them had hepatosplenomegaly. All showed atypical lymphocyte morphologic features with plasmacytoid differentiation and irregular nuclei; 3 had increased prolymphocytes. Flow cytometry demonstrated 5 patients with typical and one patient with atypical immunophenotype. All expressed ZAP70; 5 assessed patients had unmutated $I G H V$ genes. Karyotyping identified $\mathrm{t}(2 ; 14)(\mathrm{p} 16 ; \mathrm{q} 32)$ as the sole abnormality in 1 , primary abnormality in 2, and part of a complex karyotype in 3 patients. FISH analysis revealed BCL11A-IGH rearrangement in all of them. After chemotherapy, 3 patients died of disease and 3 were still alive after a median follow-up of 80 months [6].

Podgornik et al. described a 45-year-old woman with CLL that had atypical phenotype and an aggressive course; initially she had trisomy 12 as only chromosomal abnormality; she was treated with fludarabine, cyclophosphamide, and alemtuzumab with good partial response; after chemo she achieved 5-year disease-free interval; when the disease recurred she underwent an unrelated allogeneic stem cell transplant. One year later, she developed skin lesions that turned out to be Richter's transformation. Her cytogenetics showed trisomy 12 with concomitant balanced translocations $\mathrm{t}(2 ; 14)(\mathrm{p} 13 ; \mathrm{q} 32), \mathrm{t}(14 ; 19)(\mathrm{q} 32 ; \mathrm{q} 13)$, and $\mathrm{t}(18 ; 22)(\mathrm{q} 21 ; \mathrm{q} 11)$. She was successfully treated with 4 doses of ofatumumab achieving a durable remission [5].

Küppers et al. reported 2 adults and 2 pediatric CLL cases with $\mathrm{t}(2 ; 14)$ (p13;q32.3); all of these patients had unmutated IGHV genes; interestingly one patient was diagnosed with CLL clinically, but the lymph node biopsy was consistent with lymphoplasmacytic lymphoma/immunocytoma expressing monoclonal IgM. This paper focused on the technical aspects of detecting the specific location of the translocation and described that all the IGH breaks occurred within the Sy region, whilst the $2 \mathrm{p} 13$ breaks clustered centromeric of a CpG island associated with the $5^{\prime}$ end of the BCL11A gene [7].

Satterwhite et al. published 4 CLL cases with $(2 ; 14)(\mathrm{pl} 3$; q32.3), 2 of them were adults and 2 pediatric cases, and interestingly 3 cases exhibited these translocations as the sole or primary cytogenetic abnormality. The first adult patient was a 62-year-old woman, who presented with generalized lymphadenopathy, splenomegaly, and WBC of $396 \mathrm{~K} / \mu \mathrm{L}$. Cytogenetics showed 46,XX,t $(2 ; 14)(\mathrm{p} 13 ; \mathrm{q} 32)[14] / 46$,idem, $\mathrm{t}(3 ; 6)(\mathrm{p} 21 ; \mathrm{q} 25), \operatorname{del}(11)(\mathrm{q} 22 \mathrm{q} 23)[2] / 46$,idem,add(8)(p23)[2] indicating that $\mathrm{t}(2 ; 14)(\mathrm{p} 13 ; \mathrm{q} 32)$ was the primary cytogenetic abnormality [8]. She was treated with chlorambucil and subsequently with fludarabine but failed to respond to either and died of progressive disease after 2 years of diagnosis.

The second adult patient presented with generalized lymphadenopathy, hepatosplenomegaly, and a WBC of $38.3 \mathrm{~K} / \mu \mathrm{L}$. The clinical diagnosis of CLL was established. However, a lymph node biopsy was consistent with a lymphoplasmacytic immunocytoma according to the 
Kiel classification with increased proliferation activity and monoclonal IgM kappa expression. Cytogenetic analysis of both the lymph node and the peripheral blood showed the karyotype: $46, \mathrm{XY}, \mathrm{t}(2 ; 14)(\mathrm{p} 13 ; \mathrm{q} 32), \mathrm{t}(18 ; 21)(\mathrm{p} 11 ; \mathrm{q} 21)$. After 6 years, the patient presented with clinical progression including lymphadenopathy, B-symptoms, and a WBC of $76.5 \mathrm{~K} / \mu \mathrm{L}$. Histopathology of a repeat lymph node biopsy again revealed lymphoplasmacytic immunocytoma. Chromosomal analysis showed the karyotype: 46,XY,t(2;14)(p13; $\mathrm{q} 32), \mathrm{t}(18 ; 21)(\mathrm{p} 11 ; \mathrm{q} 21)[6] / 46$,idem,t(13;15)(q12;13;q21). This patient died after 13 years of the diagnosis due to progressive disease [9]. He did not describe the clinical findings of the pediatric cases.

Richardson et al. reported two cases of CLL in children with $\mathrm{t}(2 ; 14)$ (p13; q32) in 1992. He hypothesized that there may be a potential oncogene located near the $2 \mathrm{p} 13$ breakpoint which may have been activated by the $t(2 ; 14)$ translocation in these two cases of CLL [9]. Fell et al. described the first two cases of CLL in children $\mathrm{t}(2 ; 14)$ (p13;q32) opening the hypothesis that these cases represent a rare but distinct subgroup of CLL/SLL with a specific cytogenetic change [10].

\section{Conclusion}

Here we report the case of a young male with very high WBC that was found to have CLL/SLL with associated $t(2 ; 14)$ translocation (BCL11A-IGH) and trisomy 12. To the best of our knowledge, there have been only 19 cases reported in the literature; all of them had younger age compared to regular CLL patients and 8 patients were pediatric cases; most of them had atypical cytology features and unmutated immunoglobulin heavy chain mutation status and were ZAP70 positive. In addition, the majority of these patients also presented with diffuse lymphadenopathy and elevated WBC and had the $t(2 ; 14)$ translocation as the sole or primary cytogenetic abnormality. Of note, the most frequently associated chromosomal abnormalities in CLL/SLL, such as del 11q, trisomy 12, del 13q, and del 17q, were absent in these patients. Interestingly there were 2 patients that were found to have lymphoplasmacytic lymphoma/immunocytoma with this translocation. We do not have enough available long term survival data to evaluate if the presence of this gene fusion (i.e., BCL11A-IHG) has a poorer or better overall survival, but most of the reported patients have survived for several years with the standard chemotherapy, suggesting that its impact in overall survival may not be important enough to grant more aggressive therapies and/or different follow-up schemes.

\section{Competing Interests}

The authors declare that they have no competing interests.
[2] J. Li, H. Shen, K. L. Himmel et al., "Leukaemia disease genes: large-scale cloning and pathway predictions," Nature Genetics, vol. 23, no. 3, pp. 348-353, 1999.

[3] T. Nakamura, Y. Yamazaki, Y. Saiki et al., "Evi9 encodes a novel zinc finger protein that physically interacts with BCL6, a known human B-cell proto-oncogene product," Molecular and Cellular Biology, vol. 20, no. 9, pp. 3178-3186, 2000.

[4] K. Pulford, A. H. Banham, L. Lyne et al., "The BCL11AXL transcription factor: its distribution in normal and malignant tissues and use as a marker for plasmacytoid dendritic cells," Leukemia, vol. 20, no. 8, pp. 1439-1441, 2006.

[5] H. Podgornik, J. Pretnar, B. Skopec, D. Andoljšek, and P. Černelč, "Concurrent rearrangements of BCL2, BCL3, and BCL11A genes in atypical chronic lymphocytic leukemia," Hematology, vol. 19, no. 1, pp. 45-48, 2014.

[6] C. C. Yin, K. I.-C. Lin, R. P. Ketterling et al., "Chronic lymphocytic leukemia with $\mathrm{t}(2 ; 14)(\mathrm{p} 16 ; \mathrm{q} 32)$ involves the BCL11A and IgH genes and is associated With atypical morphologic features and unmutated $\operatorname{IgV} \mathrm{H}$ genes," American Journal of Clinical Pathology, vol. 131, no. 5, pp. 663-670, 2009.

[7] R. Küppers, T. Sonoki, E. Satterwhite et al., "Lack of somatic hypermutation of $I G V_{H}$ genes in lymphoid malignancies with $\mathrm{t}(2 ; 14)(\mathrm{p} 13 ; \mathrm{q} 32)$ translocation involving the BCL11A gene," Leukemia, vol. 16, no. 5, pp. 937-939, 2002.

[8] E. Satterwhite, T. Sonoki, T. G. Willis et al., "The BCL11 gene family: involvement of BCL11A in lymphoid malignancies," Blood, vol. 98, no. 12, pp. 3413-3420, 2001.

[9] A. L. Richardson, C. G. Humphries, and P. W. Tucker, "Molecular cloning and characterization of the $t(2 ; 14)$ translocation associated with childhood chronic lymphocytic leukemia," Oncogene, vol. 7, no. 5, pp. 961-970, 1992.

[10] H. P. Fell, R. G. Smith, and P. W. Tucker, "Molecular analysis of the $t(2 ; 14)$ translocation of childhood chronic lymphocytic leukemia," Science, vol. 232, no. 4749, pp. 491-494, 1986.

[11] Y. Saiki, Y. Yamazaki, M. Yoshida, O. Katoh, and T. Nakamura, "Human EVI9, a homologue of the mouse myeloid leukemia gene, is expressed in the hematopoietic progenitors and down-regulated during myeloid differentiation of HL60 cells," Genomics, vol. 70, no. 3, pp. 387-391, 2000.

[12] S. Menzel, C. Garner, I. Gut et al., "A QTL influencing F cell production maps to a gene encoding a zinc-finger protein on chromosome 2p15," Nature Genetics, vol. 39, no. 10, pp. 11971199, 2007.

[13] D. Pfeifer, M. Pantic, I. Skatulla et al., "Genome-wide analysis of DNA copy number changes and LOH in CLL using highdensity SNP arrays," Blood, vol. 109, no. 3, pp. 1202-1210, 2007.

\section{References}

[1] P. Liu, J. R. Keller, M. Ortiz et al., "Bcllla is essential for normal lymphoid development," Nature Immunology, vol. 4, no. 6, pp. 525-532, 2003. 


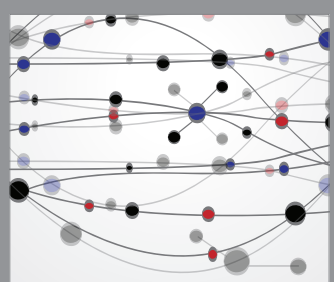

The Scientific World Journal
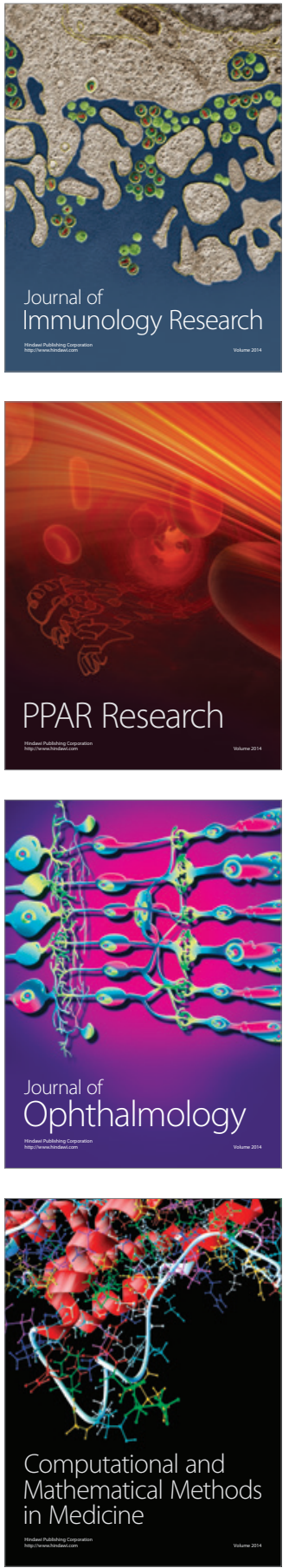

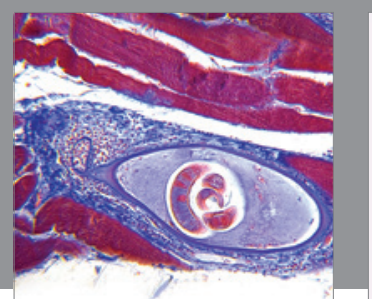

Gastroenterology Research and Practice

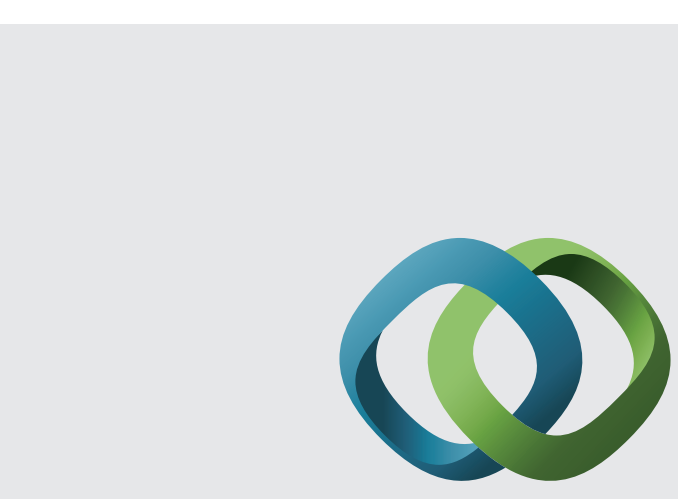

\section{Hindawi}

Submit your manuscripts at

http://www.hindawi.com
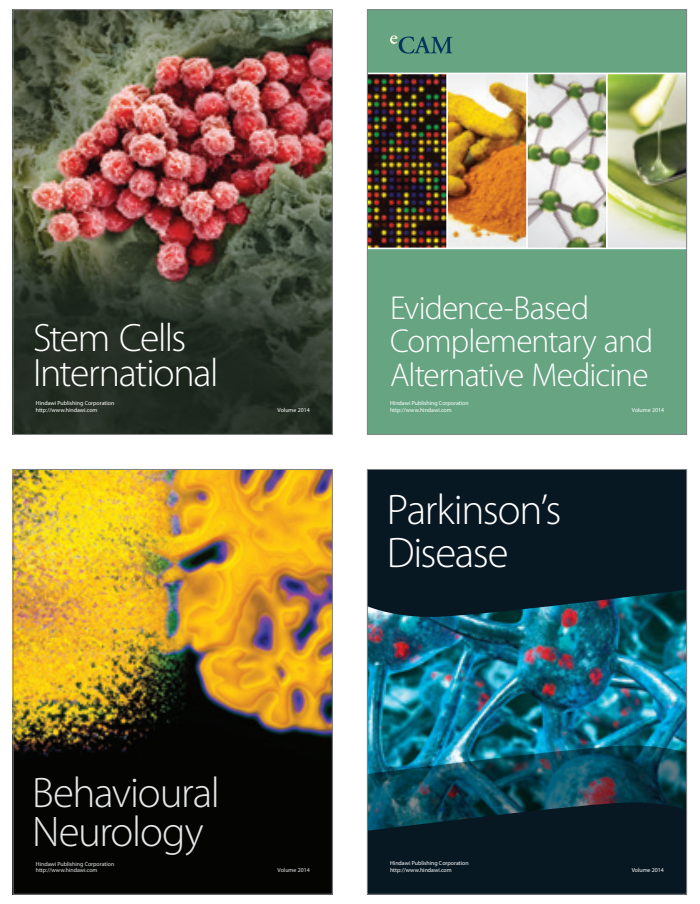
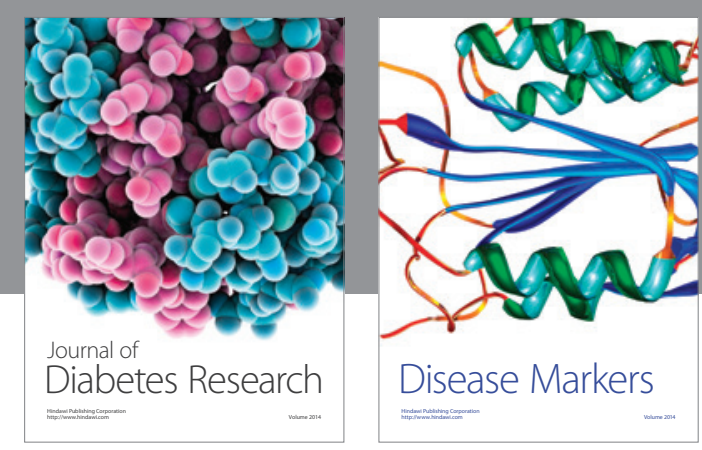

Disease Markers
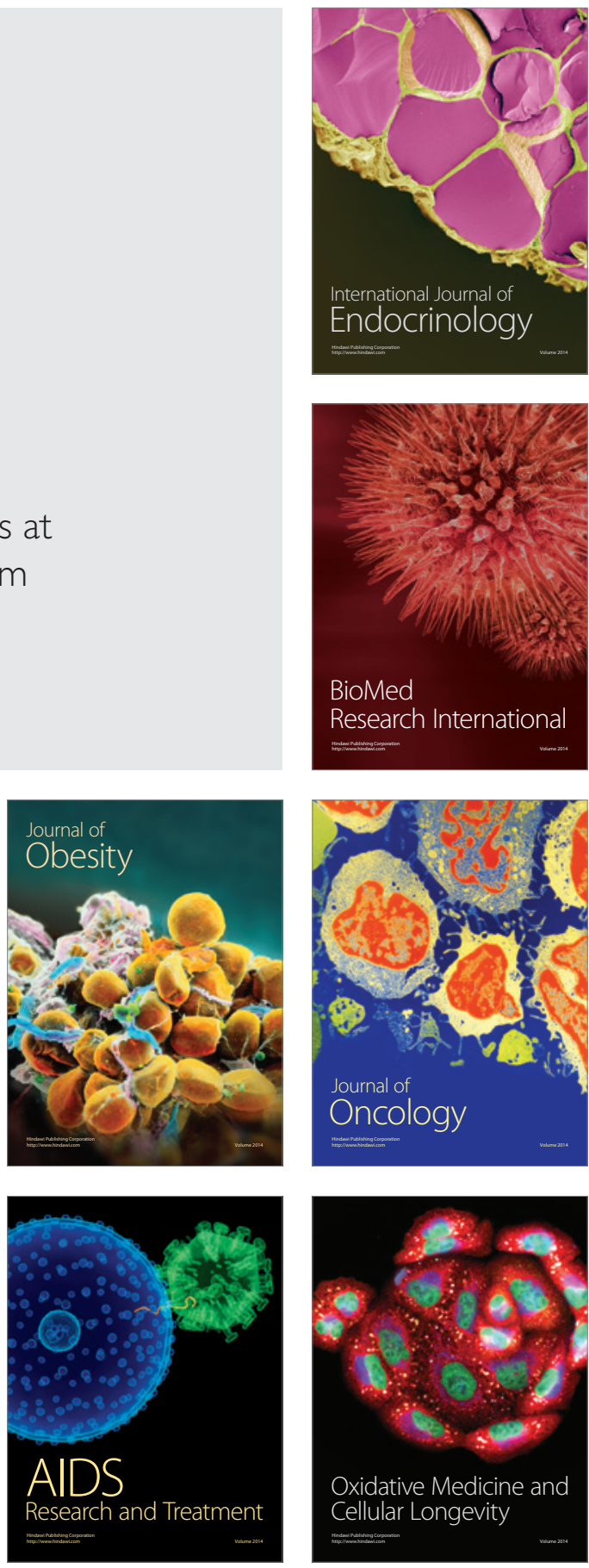